\title{
Review
}

\section{The choice and cardiovascular safety of particular nonsteroidal anti-inflammatory drugs}

\author{
Jonas Černeckis ${ }^{\mathrm{a},{ }^{*}}$, Austejja Samuolytė ${ }^{\mathrm{b}}$, Greta Baltušytė $^{\mathrm{b}}$, \\ Rugilè Ivanickaitė ${ }^{c}$, Kristijonas Puteikis ${ }^{\mathrm{d}}$ \\ a University of Cambridge, Homerton College Cambridge, United Kingdom \\ $\mathrm{b}$ Vilnius Lyceum, Vilnius, Lithuania \\ c Vilnius University, Faculty of Chemistry and Geosciences, Vilnius, Lithuania \\ d Vilnius University, Faculty of Medicine, Vilnius, Lithuania \\ Received 5 September 2017; accepted 14 February 2018
}

\begin{abstract}
Summary
Nonsteroidal anti-inflammatory drugs (NSAIDs) are authorized for the relief of pain and inflammation in a wide range of conditions including the discomfort associated with headaches, osteoarthritis, rheumatoid arthritis, and menstrual pain and are available by prescription or over-the-counter (OTC). We reviewed common adverse effects of NSAIDs (especially those related to cardiovascular [CV] system) along with NICE (National Institute for Health and Care Excellence), EMA (European Medicines Agency), and the FDA (U.S. Food and Drug Administration) guidelines for safe and effective use of particular NSAIDs. Furthermore, we examined the NSAIDs market in Lithuania in a period between June 2016 and May 2017 and discussed how well recommendations mentioned above were followed. We emphasized that there was a high percentage of diclofenac prescribed in Lithuania while international guidelines encourage prescribing ibuprofen or naproxen for their relatively lower CV risk. Reviewing past trials, we observed that despite existing guidelines no single NSAID could be considered to be the safest one due to a lack of superiority trials.
\end{abstract}

Seminars in Cardiovascular Medicine 2018; 24:16-21

Keywords: NSAID, cardiovascular risk, diclofenac safety, naproxen, NSAID guidelines

\section{Introduction}

Nonsteroidal anti-inflammatory drugs (NSAIDs) are among the most used drugs in the world because of their capability of reducing acute or chronic pain. With some being prescribed, other NSAIDs are sold over-the-counter (depending on the country). Even though NSAIDs are easily accessible, it is important to consider their possible side effects. NICE (National Institute for Health and Care Excellence), EMA (European Medicines Agency) and the FDA (U.S. Food and Drug Administration) caution doctors and patients that new studies emerge showing the negative impact of NSAIDs, especially to the cardiovascular (CV) system [1-3]. It is important to ensure that all healthcare professionals are guided by the newest international recommendations to choose treat-

\footnotetext{
* Corresponding address: Homerton College, University of Cambridge, Hills Road, CB2 8PH, Cambridge, United Kingdom. Tel.: +44 1223747111.

E-mail: jc2072@cam.ac.uk (J. Černeckis).
}

ments with the lowest risk. The purpose of this article is to review trials from the last several years as well as current international recommendations to highlight which NSAIDs could be considered to be the safest to use today and to discuss the statistics of NSAID sales in Lithuania [4].

\section{Mechanism of action and adverse effects}

\section{Mechanism of action}

NSAIDs are primarily known to be responsible for the inhibition of prostaglandin biosynthesis by inhibiting the activity of cyclooxygenase1 (COX-1) and cyclooxygenase-2 (COX-2). As COX enzymes metabolize arachidonic acid to prostaglandin $\mathrm{H}_{2}\left(\mathrm{PGH}_{2}\right)$, their inhibition hinders the use of $\mathrm{PGH}_{2}$ by $\mathrm{PG}$ isomerases as the substrate to form prostacyclin $\left(\mathrm{PGI}_{2}\right)$ and thromboxane $\mathrm{A}_{2}\left(\mathrm{TXA}_{2}\right)$. However, there are other pathways as well: some NSAIDs inhibit the lipoxygenase pathway, while others interfere with the G-protein-mediated signal transduction pathway. 
By blocking COX-2 enzymes NSAIDs relieve pain in addition to lowering fever and reducing swelling [5].

\section{Adverse effects}

Adverse drug reactions (ADRs) of NSAIDs comprise a serious public health problem as these drugs are in high demand and so are sometimes overused [6]. NSAIDs mainly affect the gastrointestinal (GI) tract, kidney function and the $\mathrm{CV}$ system; however, the magnitude of the risk for these organ systems may depend on the choice of a particular NSAID and its dosage [7]. COX-1 inhibition damages the gastric lining as the synthesis of mucosal-protective prostaglandins is inhibited: NSAIDs cause a reduction of mucus and bicarbonate secretion, as well as a reduction of blood flow in the epithelial lining. Consequently, that increases the risk of mucosal damage, epithelial damage, and ulceration [8]. Meta-analyses from a total of 639 randomized trials performed by the Coxib and traditional NSAID Trialists' (CNT) Collaboration published in 2013 noted an increase in upper GI complications with the use of all NSAIDs evaluated: the relative risk was 1.81 (95\% $\mathrm{CI}=1.17-2.81, p=0.0070)$ for $\mathrm{COX}-2$ selective inhibitors, $\mathrm{RR}=1.89(95 \% \mathrm{CI}=1.16-3.09, p=$ $0.0106)$ for diclofenac, $\mathrm{RR}=3.97(95 \% \mathrm{CI}=2.22$ 7.10, $p<0.0001$ ) for ibuprofen and $\mathrm{RR}=4.22$ $(95 \% \mathrm{CI}=2.71-6.56, p<0.0001)$ for naproxen [9]. High relative risk of GI complications for naproxen should be considered when the drug is prescribed to patients with a high risk of GI complications, but sometimes this risk might be outweighed by possible benefits of a lower risk of CV complications discussed later [10]. Although serious ADRs affecting kidneys comprise only a small percentage of patients taking NSAIDs, it is known that non-selective cyclooxygenase inhibitors can alter the glomerular filtration rate (through vasoconstriction of the afferent arteriole in the nephron), causing peripheral edema, hypertension, and kidney injury. Such renal abnormalities are particularly dangerous to patients with reduced renal blood perfusion and can induce a severe deterioration of renal functions [11]. NSAIDs can drastically affect the CV system as well: according to one previously proposed theory, the selective inhibition of COX-2 in endothelial cells of a blood vessel can cause an imbalance of prostacyclin $\left(\mathrm{PGI}_{2}\right)$ and $\mathrm{TXA}_{2}$ in the blood, resulting in vasoconstriction and platelet clumping. It was hypothesized that $\mathrm{PGI}_{2}$ along with other mediators restricted the formation of thrombus at the site of vascular injury, so the inhibition of $\mathrm{PGI}_{2}$ would result in an elevated risk of thrombotic events such as myocardial infarction (MI), stroke, or other CV events [12-14].
However, newer findings suggest that the vascular contribution of $\mathrm{PGI}_{2}$ derived from COX-2 enzymatic action is small in humans and the antithrombotic role of COX-2 in the vascular system might not be so extensive. Furthermore, renal and CV side effects could be related to each other to a greater extent than once thought, as $\mathrm{PGI}_{2}$ inhibition may foster the formation of atherosclerotic lesions both through plaque development and the distortion of blood pressure control in the kidneys (by reduction of blood flow in afferent arterioles due to the lack of $\mathrm{PGI}_{2}$ vasodilator effect) [12]. Possible mechanisms for these side effects could be investigated further to understand whether there are differences of CV risk with the use of different NSAIDs (as summarised in Table 1 and discussed next) and why they appear.

\section{CV risk associated with particular NSAIDs in the context of trials and guidelines}

\section{Diclofenac}

Diclofenac is a non-selective NSAID and is suspected to be riskier for people with predisposed CV conditions [15-17]. Several studies have found evidence that diclofenac increased the risk of major CV events. A case-control study found the risk was associated with cerebrovascular events when using diclofenac or ketoprofen [18]. Similar data holds for MI and therefore further questions the use of diclofenac: P. Ungprasert mentions a systematic review of observational studies that reveals a summary relative risk of diclofenac for MI to be very similar compared with rofecoxib ( $25 \mathrm{mg} / \mathrm{d}$ or less) which is now removed from the market due to CV safety issues $[16,19]$. He pointed to a 2011 systematic review of observational data similarly showing that the relative risk of diclofenac for stroke was similar to that of rofecoxib but no evidence for a similar association for naproxen, ibuprofen, and celecoxib was found due to a lack of data at the time of the investigation [20]. Results from the meta-analysis of randomized trials by the CNT Collaboration mentioned above showed diclofenac to be associated with major vascular events together with a COX-2 inhibitor [9]. A safety advice was issued by the European Medicines Agency (EMA) in June 2013 afterwards, stating that diclofenac should not be prescribed for patients with "established congestive heart failure (New York Heart Association (NYHA) class II-IV), ischemic heart disease, peripheral arterial disease or cerebrovascular disease." [1]. Similarly, the Medicines and Healthcare products Regulatory Agency (MHRA) has encouraged a switch to another NSAID for patients with the same conditions using diclofenac 
[21]. EMA also encourages individual evaluations for patients with $\mathrm{CV}$ risk factors, such as hypertension, hyperlipidaemia, diabetes mellitus and smoking. It also stresses the need to review patient data for those using diclofenac and to choose either an alternative NSAID or to continue diclofenac therapy at the lowest effective dose for the shortest time possible. P. McGettigan and D. Henry proposed to withdraw diclofenac from the Essential Medicines List (EML) in a data review because of the relative $\mathrm{CV}$ risk similar to the one of rofecoxib (mentioned above) [22]. A.E. Bello and R.J. Holt even questioned why the FDA did not discuss the use of diclofenac as there was evidence that diclofenac was less safe than other NSAIDs [10]. While there are studies showing diclofenac may be comparable to other NSAIDs, prescription of the drug is often discouraged for those at risk or having a history of CV events [23]. However, there is a need to note limitations of the meta-analyses mentioned above, while a non-inferiority study could help find out whether diclofenac is comparable with other NSAIDs.

\section{Naproxen}

For some time naproxen was thought to be one of the safest NSAIDs and was not considered to increase the risk of CV events [17]. A meta-analysis of data from randomized trials concluded that selective COX-2 inhibitors increased the risk of $\mathrm{CV}$ events similarly to ibuprofen and diclofenac, but researchers could not associate naproxen with such excessive risk [24]. A meta-analysis by the Coxib and traditional NSAID Trialists' (CNT) Collaboration mentioned above shows that during trials of NSAIDs versus either placebo or other NSAID, high-dose coxibs and diclofenac similarly increase the risk of major vascular (mostly coronary) events, ibuprofen significantly increases solely major coronary events, but naproxen does not [9]. S. Trelle et al. found the risk of stroke to be less associated with naproxen compared to that of ibuprofen and diclofenac [25]. P. McGettigan and D. Henry also noted naproxen to be the least harmful in a systematic review of observational studies [26]. Another nested case-control study found the OR of increased risk of being admitted to the hospital because of heart failure to be quite similar among some NSAIDs: naproxen, ibuprofen, diclofenac and rofecoxib [27]. Even though coxibs and other NSAIDs are being compared in this article, it is worth noting that coxibs have gone through fewer trials than other NSAIDs since their clinical use as several decades elapsed between the first use of older NSAIDs and the appearance of coxibs in the market. A novel notion that all NSAIDs (including naproxen) pose a threat to the CV system is now widely established, as the warning from the FDA in 2015 and two recent trials at the end of 2016 and 2017 have shown that naproxen could not be stated as the safest NSAID [2,28,29]. A recent nationwide case-time-control study in Denmark found an increased risk of out-of-hospital cardiac arrest with the use of ibuprofen and diclofenac, but lacked statistical power to evaluate the risk associated with celecoxib, naproxen, and the withdrawn rofecoxib [30]. This study pointed out that naproxen was not widely used in some countries (such as Denmark); one study in 15 countries found that naproxen composed $9.4 \%$ of the evaluated NSAID market [22]. Due to lower numbers of consumption, any information about the effects of naproxen may not be as reliable, as for more commonly used NSAIDs. Because of this and other limitations of studies (such as biases in meta-analyses) there is less of a consensus nowadays about the CV safety of naproxen. A recent systematic review of four studies and bayesian meta-analysis by $\mathrm{M}$. Bally et al. found that the risk of MI increases with the use of any NSAID selected: celecoxib, ibuprofen, diclofenac, naproxen, and rofecoxib [28]. Prior research to the one of M. Bally et al., PRECISION, a randomized, double-blind non-inferiority trial, found that celecoxib, ibuprofen and naproxen all similarly increase the risk of $\mathrm{CV}$ events and some of the guidelines published later were based on PRECISION to state the three NSAIDs (celecoxib, ibuprofen, and naproxen) as comparable [29]. These two studies did not conclude the superiority of naproxen due to their limitations. Guidelines for NSAID prescriptions do not currently indicate clear preference towards naproxen as well. The FDA, for instance, strengthened their warning for the use of all NSAIDs in 2015 and stressed that no NSAID could be regarded to be the safest one due to a lack of superiority trials [2]. It is for the GI side effects that the FDA, according to A.E. Bello and R.J. Holt, did not exclude naproxen from their warning: after voting 16:9 against the notion that the existing data favours naproxen due to a lower $\mathrm{CV}$ effects risk, some members acknowledged to have voted due to common GI side effects related to naproxen. GI side effects are the reason why naproxen could not be perceived as the most beneficial of common NSAIDs; the potential (but unproven) CV benefit has to be further investigated [10].

\section{EMA and NICE guidelines for the prescription of NSAIDs}

If a patient needs a NSAID, NICE recommends prescription of ibuprofen (up to $1200 \mathrm{mg} / \mathrm{d}$ ) or 
Table 1.

Reviewed study data for increased risk of cardiovascular complications when using NSAIDs; RR - relative risk, RRR - ratio of relative risks, $\mathrm{OR}$ - odds ratio, $\mathrm{HR}$ - hazard ration, $\mathrm{CI}$ - confidence interval

\begin{tabular}{|c|c|c|c|c|c|}
\hline Studies & $\begin{array}{l}\text { Publication } \\
\text { year }\end{array}$ & Type of study & Study end points & $\begin{array}{c}\text { Number of } \\
\text { patients or } \\
\text { cases } \\
\text { (studies } \\
\text { reviewed) }\end{array}$ & $\begin{array}{l}\text { Odds ratios ( } 95 \% \text { confidence } \\
\text { interval) }\end{array}$ \\
\hline
\end{tabular}

\begin{tabular}{lcll}
\hline $\begin{array}{l}\text { Nissen SE, } \\
\text { Yeomans ND, } \\
\text { et al. [29] }\end{array}$ & 2016 & $\begin{array}{l}\text { Randomized, } \\
\text { double-blind } \\
\text { noninferiority } \\
\text { trial }\end{array}$ & $\begin{array}{l}\text { Cardiovascular } \\
\text { events }\end{array}$ \\
$\begin{array}{l}\text { Kearney PM, } \\
\text { Baigent C, et } \\
\text { al. [24] }\end{array}$ & 2006 & $\begin{array}{l}\text { Meta-analysis of } \\
\text { randomized trials }\end{array}$ & $\begin{array}{l}\text { Cardiovascular } \\
\text { events }\end{array}$ \\
& & & \\
$\begin{array}{l}\text { Bhala N, } \\
\text { Emberson J, } \\
\text { et al. [9] }\end{array}$ & 2013 & $\begin{array}{l}\text { Meta-analysis of } \\
\text { randomized trials }\end{array}$ & $\begin{array}{l}\text { Major vascular } \\
\text { events }\end{array}$
\end{tabular}

reviewed)

Son randomized trials events

145373

$24081 \quad$ Celecoxib versus naproxen $\mathrm{HR}=0.90(95 \% \mathrm{CI}=0.71-1.15)$; celecoxib vs. ibuprofen $\mathrm{HR}=$ $0.81(95 \% \mathrm{CI}=0.65-1.02)$

COX-2 inhibitors $\mathrm{RR}=1.42$ $(95 \% \mathrm{CI}=1.13-1.78)$; ibuprofen $\mathrm{RR}=1.51(95 \% \mathrm{CI}=0.96-2.37)$; diclofenac $\mathrm{RR}=1.63(95 \% \mathrm{CI}=$ 1.12-2.37); naproxen $R R=0.92$ $(95 \%$ CI $=0.67-1.26)$ (compared with placebo) Diclofenac $\mathrm{RR}=1.41(95 \% \mathrm{CI}=$ 1.12-1.78); COX-2 inhibitor $\mathrm{RR}=1.37(95 \% \mathrm{CI}=1.14-1.66)$; naproxen $\mathrm{RR}=0.93(95 \% \mathrm{CI}=$ 0.69-1.27); ibuprofen $\mathrm{RR}=1.44$ $(95 \% \mathrm{CI}=0.89-2.33)$ and $\mathrm{RR}=$ $2.22(95 \% \mathrm{CI}=1.10-4.48)$ for major coronary events (compared with placebo)

Trelle S, Reichenbach S, et al. [25]

Bally M, Dendukuri N, et al. [28]

\section{McGettigan P, Henry D [19] McGettigan P, Henry D [26]}

Arfè A, Scotti L, et al. [27]

Lapi F, Piccinni C, et al. [18] Sondergaard $\mathrm{KB}$, Weeke $\mathrm{P}$, et al. [30]
2011 Network Stroke

meta-analysis

2017

Systematic review of and bayesian

Myocardial meta-analysis

Systematic review of observational studies Systematic review of observational studies

Myocardial infarction

$86193(23)$

Major vascular events

2016

Nested

case-control study

Risk of hospital admission for heart failure

\section{6}

Case-control

Cerebrovascular event

2016

Case-time-control

Out-of-hospital cardiac arrest
Naproxen RR $=1.76(95 \% \mathrm{CI}=$ 0.91-3.33); ibuprofen $\mathrm{RR}=3.36$ $(95 \% \mathrm{CI}=1.00-11.60)$; diclofenac $\mathrm{RR}=2.86(95 \% \mathrm{CI}=$ 1.09-8.36)

446763 (4) $\quad$ Celecoxib, $\mathrm{OR}=1.24(95 \% \mathrm{CI}=$ 0.91-1.82); ibuprofen $\mathrm{OR}=1.48$ (95\% CI = 1.00-2.26); diclofenac $\mathrm{OR}=1.50(95 \% \mathrm{CI}=$ 1.06-2.04); naproxen $\mathrm{OR}=1.53$ $(95 \% \mathrm{CI}=1.07-2.33)$; rofecoxib $\mathrm{OR}=1.58(95 \% \mathrm{CI}=1.07-2.17)$

Diclofenac RR $=1.40(95 \% \mathrm{CI}=$ 1.16-1.70); rofecoxib $\mathrm{RR}=1.33$ $(95 \% \mathrm{CI}=1.00-1.79)$

Naproxen 1.09 (95\% CI = 1.02-1.16); ibuprofen RRR = $1.18(95 \% \mathrm{CI}=1.11-1.25)$; diclofenac $\mathrm{RRR}=1.40(95 \% \mathrm{CI}=$ 1.27-1.55); rofecoxib RRR = $1.45(95 \% \mathrm{CI}=1.33-1.59)$

92163 Naproxen OR $=1.16(95 \%$ CI $=$ 1.07-1.27); ibuprofen $\mathrm{OR}=1.18$ $(95 \% \mathrm{CI}=1.12-1.23)$; diclofenac $\mathrm{OR}=1.19(95 \% \mathrm{CI}=$ $1.15-1.24)$; rofecoxib $\mathrm{OR}=1.36$ $(95 \% \mathrm{CI}=1.28-1.44)$

29722 Diclofenac OR $=1.53(95 \% \mathrm{CI}=$ 1.04-2.24); ketoprofen $\mathrm{OR}=$ $1.62(95 \% \mathrm{CI}=1.02-2.58)$;

28947 Ibuprofen OR $=1.31(95 \% \mathrm{CI}=$ 1.14-1.51); diclofenac $\mathrm{OR}=$ $1.50(95 \% \mathrm{CI}=1.23-1.82)$ 
Table 2.

Adapted guidelines that are currently presented by EMA, NICE, and the reference 10; GI - gastrointestinal, CV - cardiovascular, PPI - proton pump inhibitor, H2RA - histamine $\mathrm{H}_{2}$ receptor antagonist

\begin{tabular}{|c|c|}
\hline Condition & Possible management/specific guidelines \\
\hline No risk of GI or CV complications [10] & Ibuprofen or naproxen as first choice, lowest effective dose \\
\hline Hightened risk of GI complications, no or low CV risk [10] & Ibuprofen or celexocib as first choice (+ PPI/H2RA if required) \\
\hline High CV risk [10] & Consider naproxen (+ low dose aspirin) \\
\hline $\begin{array}{l}\text { High CV and GI risk, frail individuals, adults with fractures, } \\
\text { low back pain or arthritis }[10,34,35]\end{array}$ & Avoid NSAIDs (if used, use with low dose aspirin and PPI) \\
\hline Allergy to NSAIDs [33] & Consider COX-2 inhibitors, inform about risks \\
\hline Severe allergic reaction to NSAIDs [33] & Consider COX-2 inhibitors (first taken in specialist setting) \\
\hline $\begin{array}{l}\text { Using renin-angiotensin drugs or with chronic kidney } \\
\text { disease [36] }\end{array}$ & Monitoring (e.g. glomerular filtration rate) required \\
\hline
\end{tabular}

naproxen (up to $1000 \mathrm{mg} / \mathrm{d}$ ) with the lowest effective dose for the shortest time possible [3]. EMA encourages using the minimal effective dose of ibuprofen as well, as $2400 \mathrm{mg} / \mathrm{d}$ and more are considered unsafe $[31,32]$. EMA also points out that proper investigation is required for patients with CV risk factors, such as smoking, hypertension, or obesity and high doses of ibuprofen are discouraged. The diclofenac is not considered an appropriate choice due to its greater CV side effects for patients with established congestive heart failure, ischaemic heart disease, peripheral arterial disease, or cerebrovascular disease and risk factors mentioned above (smoking, hypertension, hyperlipidaemia, diabetes mellitus). NICE has published guidelines for specific cases when NSAIDs are required and their shortened summary is presented in Table 2 .

\section{The correlation of sales of NSAIDs with current guidelines}

A 2013 analysis from 15 different countries showed a median of $27.8 \%$ for diclofenac in the market expressed as percent of total NSAID sales, $11 \%$ for ibuprofen, $9.4 \%$ for naproxen, and $7.2 \%$ for celecoxib [22]. The study highlighted that two drugs that were less safe in authors' opinion (diclofenac and etoricoxib) comprised 33.2\% of the market investigated. As diclofenac is not recommended as first choice in current guidelines mentioned above, it could be replaced by ibuprofen, naproxen, or celecoxib if the price of the latter is acceptable [3]. Even though these guidelines present their judgement that diclofenac could be relatively more harmful, Lithuanian sales statistics show that diclofenac comprises the highest percentage of the market from the data (adjusted as days of treatment) from June 2016 to May 2017: 1.09\% for celecoxib, 35.35\% for diclofenac, $22.79 \%$ for ibuprofen, $2.64 \%$ for naproxen, and $25.43 \%$ for naproxen and ibuprofen combined
[4]. These numbers do not seem to comply well with international recommendations that encourage to lower the prescriptions of diclofenac (EMA and NICE), as the sales levels of diclofenac comprise one-third of NSAIDs market in Lithuania. Furthermore, ibuprofen and naproxen recommended in the guidelines comprise only a quarter of the market of NSAIDs in Lithuania $[3,4]$. It is noteworthy that prescribing celecoxib is currently acceptable as well after the recent PRECISION trial (though substantial certainty is lacking), but in some situations it might be more expensive than naproxen or ibuprofen, so the price should be considered as well: in Lithuania it comprises a minimal part of the market.

\section{Conclusion}

After reviewing various trials of the last several years, we observe that the most recent data favors the notion that no NSAID could be considered the safest due to renal, GI and, most noticeably, $\mathrm{CV}$ side effects. According to some studies and recent guidelines, the $\mathrm{CV}$ safety of diclofenac is considered to be the lowest among the most common NSAIDs $[3,10]$. Even though one other drug, naproxen, is currently accepted to be comparable with other NSAIDs as having a potentially similar risk for the CV system, naproxen is recommended to be prescribed as first-line therapy for mild to moderate pain (ibuprofen being an equivalent alternative). A superiority trial is recommended to decide whether naproxen is safer than other available NSAIDs (as thought previously), but until then its prominent GI and CV side effects should be considered, especially in specific cases with risk factors or past CV events or GI complaints. 


\section{References}

[1] European Medicines Agency. New safety advice for diclofenac 2013;2013.

[2] U. S. Food and Drug Administration. FDA strengthens warning that non-aspirin nonsteroidal anti-inflammatory drugs (NSAIDs) can cause heart attacks or strokes. 2015.

[3] National Institute for Health and Care Excellence. Non-steroidal anti-inflammatory drugs. NICE Guideline (KTT13) 2017.

[4] PLLC "SoftDent". Drug sales data 2015-2017. 2017.

[5] Borazan NH, Furst DE. Nonsteroidal Anti-Inflamatory Drugs, Disease-Modifying Antirheumatic Drugs, Nonopioid Analgesics, \& Drugs Used in Gout. In: Katzung BG, Trevor AJ, editors. Basic \& Clinical Pharmacology, 13th Ed. McGraw-Hill Medical; 2015; p. 618-641.

[6] Mullan J, Weston KM, Bonney A, Burns P, Mullan J, Rudd R. Consumer knowledge about over-the-counter NSAIDs: they don't know what they don't know. Aust Nz J Publ Heal 2017;41(2):210-214.

[7] Fine M. Quantifying the impact of NSAID-associated adverse events. Am J Manag C 2013; 9(14 Suppl):s267-72.

[8] Sinha M, Gautam L, Shukla PK, Kaur P, Sharma S, Singh TP. Current perspectives in NSAID-induced gastropathy. Mediat Inflamm 2013;2013.

[9] Bhala N, Emberson J, et al. Vascular and upper gastrointestinal effects of non-steroidal anti-inflammatory drugs: meta-analyses of individual participant data from randomised trials. The Lancet 2013;382(9894):769-779.

[10] Bello AE, Holt RJ. Cardiovascular risk with non-steroidal anti-inflammatory drugs: clinical implications. Drug Safety 2014;37(11):897.

[11] Perazella MA, Luciano RL. Review of select causes of druginduced AKI. Expert Rev Clin Phar 2015;8(4):367-371.

[12] Grosser T, Ricciotti E, FitzGerald GA. The Cardiovascular Pharmacology of Nonsteroidal Anti-Inflammatory Drugs. Trends Pharmacol Sci 2017;38(8):733-748.

[13] Gunter BR, Butler KA, Wallace RL, Smith SM, Harirforoosh S. Non-steroidal anti-inflammatory drug-induced cardiovascular adverse events: a meta-analysis. J Clin Pharm Ther 2016;42(1):27-38.

[14] Anwar A, Anwar IJ, Delafontaine P. Elevation of cardiovascular risk by non-steroidal anti-inflammatory drugs. Trends Cardiovas Med 2015;25(8):726-735.

[15] Patrono C. Cardiovascular effects of nonsteroidal antiinflammatory drugs. Curr Cardiol Rep 2016;18(3):25-25.

[16] Ungprasert P. NSAIDs and cardiovascular disease: time to say no to diclofenac. Intern Emerg Med 2016;11(1):1.

[17] Danelich IM, Wright SS, Lose JM, Tefft BJ, Cicci JD, Reed BN. Safety of nonsteroidal antiinflammatory drugs in patients with cardiovascular disease. Pharmacotherapy 2015;35(5):520-535.

[18] Lapi F, Piccinni C, et al. Non-steroidal anti-inflammatory drugs and risk of cerebrovascular events in patients with osteoarthritis: a nested case-control study. Intern Emerg Med 2016;11(1):49.

[19] McGettigan P, Henry D. Cardiovascular risk and inhibition of cyclooxygenase. JAMA 2006;296(13):1633-44.
[20] Varas-Lorenzo C, Riera-Guardia N, et al. Stroke risk and NSAIDs: a systematic review of observational studies. Pharmacoepidem Dr S 2011;20(12):1225-1236.

[21] Medicines and Healthcare products Regulatory Agency. Diclofenac: new contraindications and warnings. 2013.

[22] McGettigan P, Henry D. Use of non-steroidal antiinflammatory drugs that elevate cardiovascular risk: an examination of sales and essential medicines lists in low-, middle-, and high-income countries. PLoS Med 2013;10(2):e1001388.

[23] van Walsem A, Pandhi S, et al. Relative benefit-risk comparing diclofenac to other traditional non-steroidal anti-inflammatory drugs and cyclooxygenase- 2 inhibitors in patients with osteoarthritis or rheumatoid arthritis: a network meta-analysis. Arthritis Res Ther 2015;17(1):66.

[24] Kearney PM, Baigent C, et al. Do selective cyclo-oxygenase-2 inhibitors and traditional non-steroidal anti-inflammatory drugs increase the risk of atherothrombosis? Meta-analysis of randomised trials. BMJ 2006;332(7553):1302-1308.

[25] Trelle S, Reichenbach S, et al. Cardiovascular safety of non-steroidal anti-inflammatory drugs: network meta-analysis. BMJ 2011;342:c7086.

[26] McGettigan P, Henry D. Cardiovascular risk with non-steroidal anti-inflammatory drugs: systematic review of population-based controlled observational studies. PLoS Med 2011; 8(9):e1001098.

[27] Arfè A, Scotti L, et al. Non-steroidal anti-inflammatory drugs and risk of heart failure in four European countries: nested case-control study. BMJ 2016;354:i4857.

[28] Bally M, Dendukuri N, et al. Risk of acute myocardial infarction with NSAIDs in real world use: Bayesian meta-analysis of individual patient data. BMJ 2017;357:j1909.

[29] Nissen SE, Yeomans ND, et al. Cardiovascular safety of celecoxib, naproxen, or ibuprofen for arthritis. New Engl J Med 2016;375:2519-2529.

[30] Sondergaard KB, Weeke P, et al. Non-steroidal antiinflammatory drug use is associated with increased risk of out-of-hospital cardiac arrest: a nationwide case-time-control study. Eur Heart J-Card Pharm 2016;3(2):100-107.

[31] European Medicines Agency. Updated advice on use of high-dose ibuprofen 2015. 2015.

[32] European Medicines Agency. PRAC recommends updating advice on use of high-dose ibuprofen. 2015.

[33] National Institute for Health and Care Excellence. Drug allergy: diagnosis and management. NICE Guideline (CG183) 2014

[34] National Institute for Health and Care Excellence. Fractures (non-complex): assessment and management. NICE Guideline (NG38) 2016;1.1.6:4.

[35] National Institute for Health and Care Excellence. Low back pain and sciatica in over 16s: assessment and management. NICE Guideline (NG59) 2016.

[36] National Institute for Health and Care Excellence. Chronic kidney disease in adults: assessment and management. NICE Guideline (CG182) 2015. 